Historic, Archive Document

Do not assume content reflects current scientific knowledge, policies, or practices. 

CONYERS B. FLEU, JR.

\author{
SEEDS
}

ROSS AND MONTANA STREETS

GERMANTOWN, PHILA., PA.

U. S. A.

May 1326

SURPLUS TIST

TREE, SHRUB, EVERGREEN, PALM SEEDS

SPECIAL 10\% DISCOUNT - CASH WITH ORTER

All of the seeds offered rre fresh and of the very best quality. The season is cool and iderl for planting. Wire ordor collect or use this as an order sheet and mail at once. The seeds rie on hand and can be sent upon receipt of your oraer.

SPECIAT 10\% DISCOUNT - CASH WITH ORDER

\section{Your Name}

\section{Adaress}

Quan- Quan-

tity tity

de- on

sired hard

PASM SEED

$5 \frac{1}{2} z_{\text {. Chamaerops Excelse }}$

300 seed Cocos Australis

$325 "$ " " Plumose

$1500 "$ "Wecteliana

10 oz. Iracaena Indivisa

1300 sead Kentia Forsteriana

$1300 "$ "thounix Cinarionsis

3000 "Istania Borbonica EVERGREENS

6 1b. Abies Brachyphylla

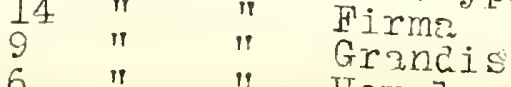

6 " " Homolepis

45 " " " " Vordmanniana

10,000 seed

10,000 seed Araucaria Imbricata

$\frac{1}{2}$ 1b. Arctostaphylos GIaca ". 50

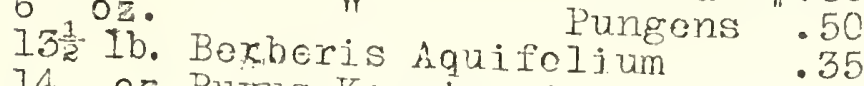

14 oz. Buxus Koreicnsis

14 1b. Cedrus it in 250 ica

250 " " " Dלodara

8 11 " $"$ " " Tibani

${ }^{2}$ " Cephalotaxus Drupacea

2 "Chamecyparis Obtusa

2 "Cinnamomum Camphors

" "Crataegus Pyrocontha

$\begin{array}{ll}\frac{1}{1} & " \text { Cryptomeria Jopon } \\ l & \text { " Cupressus Glabra } \\ l \frac{1}{4} & \text { " }\end{array}$

1" " " " "lovonions

165

$\begin{array}{ccc}165 & " \text { " Sempervirens } \\ \frac{1}{3} \frac{1}{2} " & \text { "Ier Aquifolium }\end{array}$

Juniperus Chinensis

Communis

Formosana

Prohyphylnen

Prostrata

Rigide.

Scopulorum Virgini:uns

5 oz. Falmia Latifolia

20 Ib. Iarix Dahurica

l14 " " Teptolepsis

.35
1.06

.35

.50

.35

.35

.35

.25

.15

.30

.50

.50

.35

.25

.25

.25

.35

.25

.50

.25

.25
.25

.50

.50
.25

.25
.75

75
.75

.75
.50

.50

" $"$ Laurocerasus Carolinimnr. I5

3 "Leurus Irobilis

21. "Libocerrus Decurrens $\quad .25$

$2 \frac{1}{4} "$ PhotinjaArbutifolia $\quad .25$

$10 \mathrm{oz}$. Picea A.janonsis 1.00

5 Ib. " " conadensis $(A 1 b s) .75$

$25 "$ " Excelsa $\quad .75$
ALL OFFERS MADE

SUBJECT TO GOODS BEING UNSOLD 

- page two-

\begin{tabular}{|c|c|c|c|c|c|c|c|c|}
\hline $\begin{array}{l}\text { uar- } \\
\text { ity } \\
\text { e- } \\
\text { ired }\end{array}$ & $\begin{array}{l}\text { Quan- } \\
\text { tity } \\
\text { on } \\
\text { hand }\end{array}$ & Kind of Seed & $\begin{array}{l}\text { per } \\
0 z \text {. }\end{array}$ & $\begin{array}{c}\text { per } \\
\quad \text { Ib. } \\
\end{array}$ & $\begin{array}{l}51 . k . \\
\text { price } \\
\text { per } 1 b .\end{array}$ & $\begin{array}{l}101 \mathrm{~b} . \\
\text { orice } \\
\text { per } 1 \mathrm{~b} \text {. }\end{array}$ & $\begin{array}{l}25 \text { Ib. } \\
\text { price } \\
\text { per Iu }\end{array}$ & $\begin{array}{l}\text { the } \\
\text { - Int }\end{array}$ \\
\hline $\begin{array}{l}1 \\
8 \\
1 \\
5 \\
1 \\
1 \\
3 \\
2 \\
7 \\
3 \\
4 \\
5 \\
3 \\
7 \\
5 \\
5 \\
3 \\
8 \\
7 \\
5 \\
3\end{array}$ & 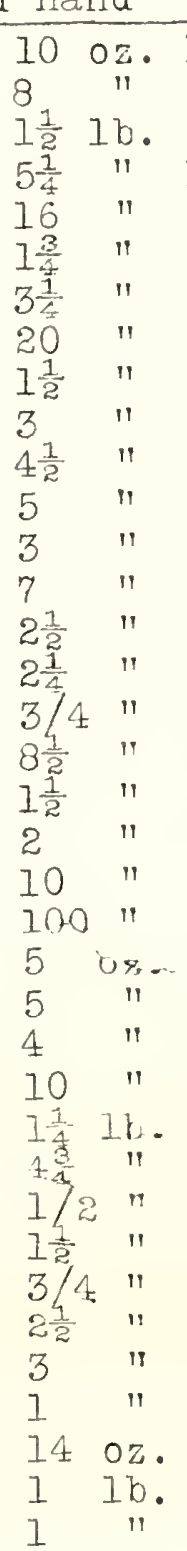 & 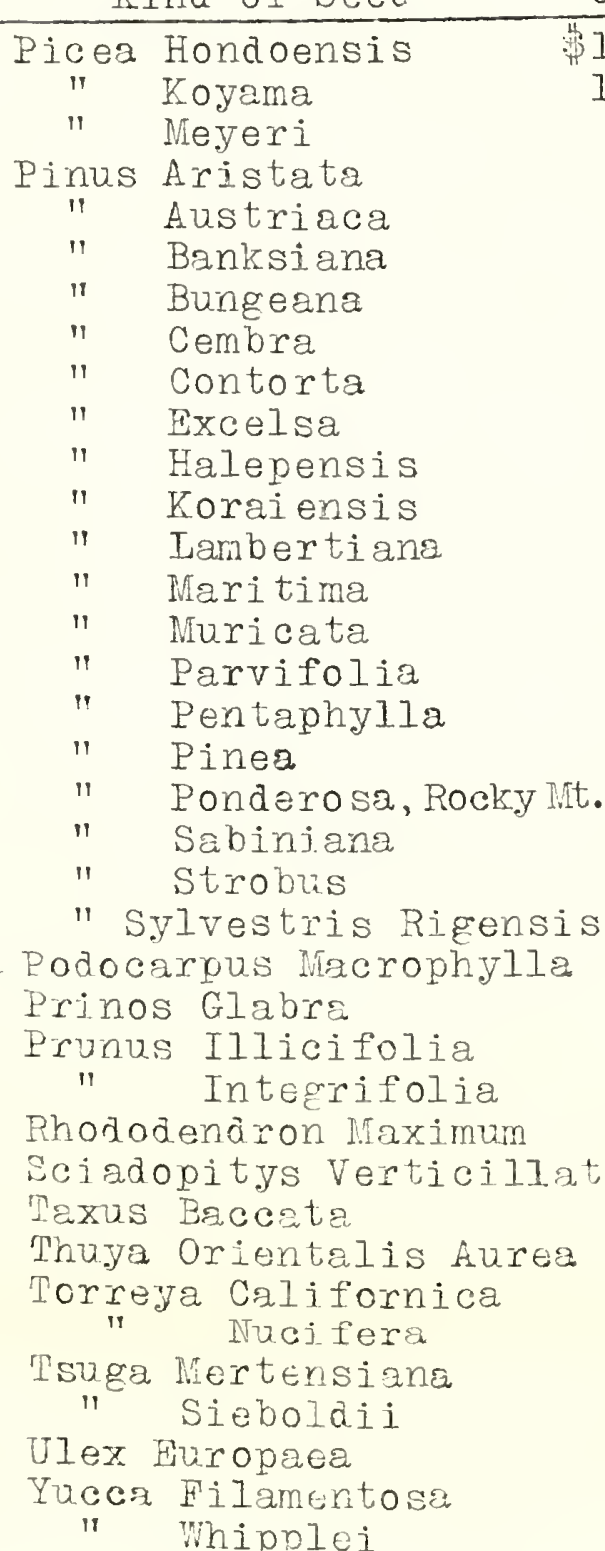 & $\begin{array}{r}1.00 \\
1.00 \\
.75 \\
.75 \\
.25 \\
.50 \\
.50 \\
.25 \\
.75 \\
.35 \\
.25 \\
.25 \\
.25 \\
.15 \\
.50 \\
.50 \\
.50 \\
.15 \\
.25 \\
.25 \\
.50 \\
.50 \\
.25 \\
.25 \\
.25 \\
.25 \\
.75 \\
.250 \\
.25 \\
.35 \\
.25 \\
.25 \\
1.00 \\
.75 \\
.50 \\
.30 \\
.50\end{array}$ & $\begin{array}{r}4.00 \\
7.50 \\
2.50 \\
5.00 \\
5.00 \\
2.00 \\
7.50 \\
3.50 \\
2.50 \\
2.50 \\
2.50 \\
1.50 \\
6.50 \\
5.00 \\
\\
1.25 \\
2.50 \\
1.75 \\
4.50 \\
4.00 \\
\end{array}$ & $\begin{array}{l}4.35 \\
3.75\end{array}$ & 1.50 & 苏1.25 & $\begin{array}{r}7.50 \\
6.00 \\
7.50 \\
26.25 \\
32.00 \\
8.50 \\
14.00 \\
25.00 \\
10.00 \\
10.00 \\
9.00 \\
11.25 \\
6.75 \\
8.7 \\
15.00 \\
10.0 \\
3.75 \\
8.50 \\
3.50 \\
3.00 \\
42.50 \\
350.0 \\
1.0 \\
70\end{array}$ \\
\hline
\end{tabular}

DRCIDUOUS TREES AND SHRUBS

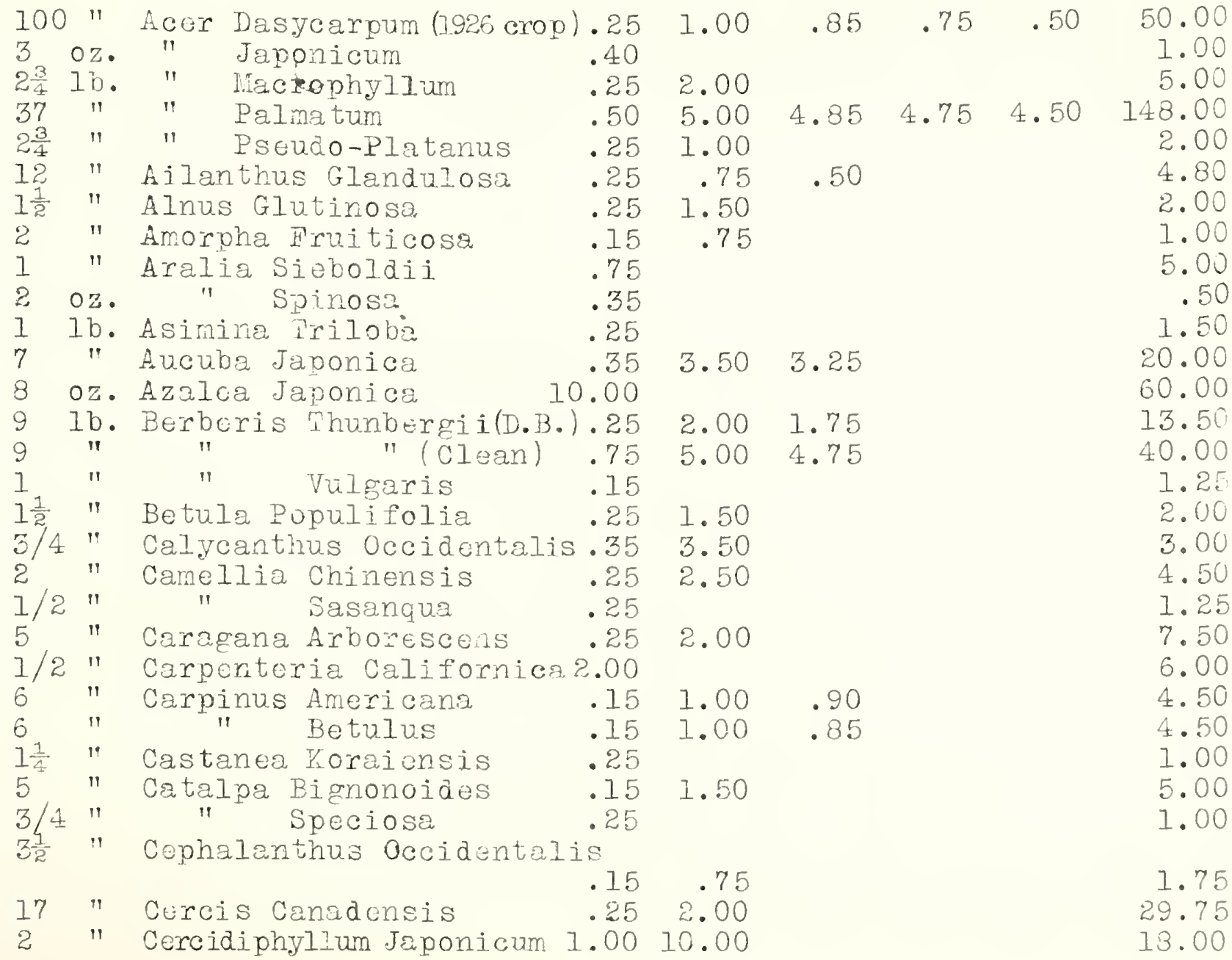





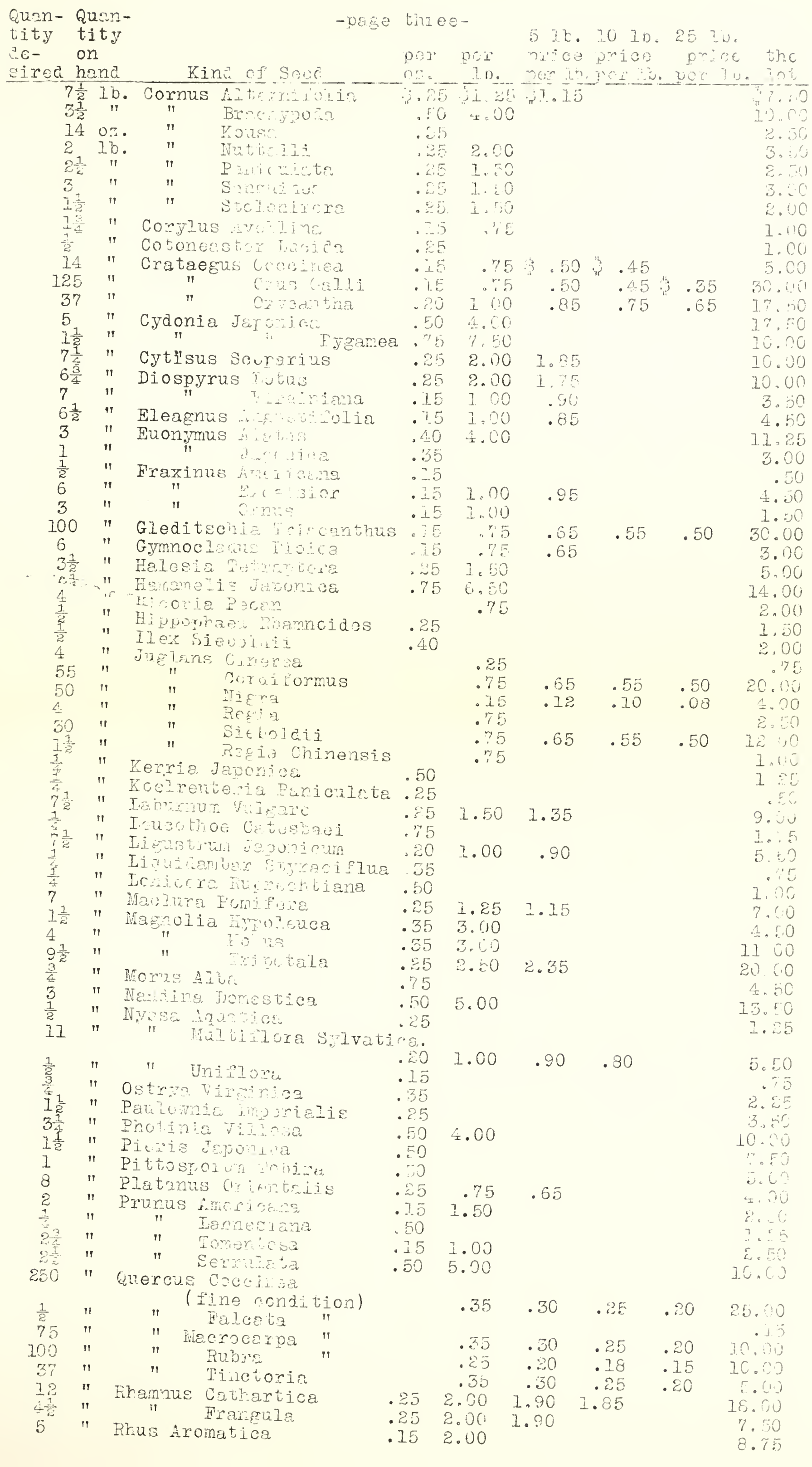





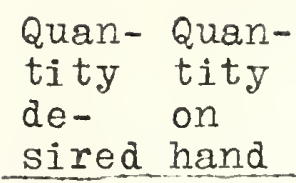

-page four-

Kind of Seod

\begin{tabular}{|c|c|c|c|c|c|c|c|c|}
\hline & $\mathrm{Ib}_{" \prime}$ & $\begin{array}{l}\text { Rhus Copalinira } \\
\text { " Cotinus (1924 crop) }\end{array}$ & $\begin{array}{r}\text { W. } 15 \\
.35\end{array}$ & $\begin{array}{r}31.00 \\
3.50\end{array}$ & $\begin{array}{l}\$ .90 \\
3.35\end{array}$ & & & 35.5 \\
\hline & $"$ & " Glabra & .15 & .75 & & & & $1 . c n$ \\
\hline $\begin{array}{l}00 \\
1\end{array}$ & oz. & $\begin{array}{l}\text { Robinia Dour-acacia } \\
\text { Romneya CuIteri }\end{array}$ & $\begin{array}{r}.15 \\
1.00\end{array}$ & 1.00 & .85 & $\$ .75$ & 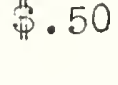 & $\begin{array}{r}30,0 ? \\
6.2 .7\end{array}$ \\
\hline & Ib. & & .15 & 1.25 & 1.15 & & & 11.0 \\
\hline & $"$ & $\begin{array}{ll}\text { " Canina } \\
\text { " Carolina }\end{array}$ & .15 & & & & & 1.00 \\
\hline & " & $\begin{array}{l}\text { Carolina } \\
\text { "Multiflora Japonica }\end{array}$ & .15 & $\begin{array}{l}1.50 \\
2.50\end{array}$ & & & & $\begin{array}{l}5.60 \\
4.50\end{array}$ \\
\hline & $"$ & " Setigera & .15 & 1.50 & 1.35 & 1.25 & & 12.03 \\
\hline & " & Salisburia Mäiantifolia & .15 & 1.00 & .85 & .80 & & 9.90 \\
\hline & $"$ & Sambucus Canadensis & .15 & 1.00 & & & & \\
\hline & $"$ & Sassafras Ufficinale & .15 & & & & & c \\
\hline & $"$ & & .15 & & & & & \\
\hline & $"$ & $\begin{array}{l}\text { Skimmia Japonica } \\
\text { Sorbus Aucuraria }\end{array}$ & $\begin{array}{r}.50 \\
.20\end{array}$ & 7.25 & 7.75 & 7.70 & 7.00 & $\begin{array}{r}2.50 \\
75.00\end{array}$ \\
\hline & $"$ & Sterculia Platanifolia & .25 & 1.00 & & $\perp . \perp v$ & 1.00 & 2.50 \\
\hline & $"$ & Symphoricarpos Rccemosu & 1.5 .25 & & & & & \\
\hline & $"$ & Svringa " Vulgaris & .25 & 1.00 & & & & 50) \\
\hline & $"$ & $\begin{array}{l}\text { SJringa Japonica } \\
\text { Vulgaris (clean }\end{array}$ & .75 & $\begin{array}{l}7.50 \\
5.00\end{array}$ & & & 4.50 & 400 \\
\hline & $"$ & Thea Sinerisis & .25 & 2.00 & 4.00 & 4.75 & 4.50 & $\begin{array}{r}400.00 \\
3.50\end{array}$ \\
\hline & $"$ & Tilia Argentea & .15 & 1.50 & 1.35 & 1.25 & & 50 \\
\hline & $"$ & "Platyonylios & .15 & 1.50 & & & & 6.25 \\
\hline & " & Viburnum Acorifolium & .15 & 1.00 & .90 & .85 & .75 & 15.00 \\
\hline & $"$ & " Dontatum & .15 & .75 & & & & 50 \\
\hline & $"$ & Iuntana & .15 & 1.50 & 1.35 & 1.25 & 1.00 & \\
\hline & $n$ & Lentago & .15 & 1.50 & 1.35 & 1.25 & & 15 \\
\hline & 0 . & " Prunitolium & .15 & & & .85 & & \\
\hline & $\mathrm{ib}$. & Tinus & .30 & & & & & \\
\hline & & VIIVES AIJ CIIIBER & & & & & & \\
\hline & $1 \mathrm{~b}$ & Actiniāia Arguta & 1.00 & & & & & 10.00 \\
\hline & $\|$ & Akebia Quinata & .40 & 4.00 & & & & \\
\hline & oz. & Celastrus Articulatus & .50 & & & & & 5 \\
\hline & Ib. & " Scanủens & .15 & 1.25 & & & & 2 \\
\hline & Oz. & Clematis Coccinea & 1.00 & & & & & 0 \\
\hline & ID. & $\begin{array}{ll}\text { NIammula } \\
\text { Vitalba }\end{array}$ & $\begin{array}{l}.25 \\
.25\end{array}$ & & & & & 10 \\
\hline & oz. & Hyarangea Petiolaris & .75 & & & & & .7 \\
\hline & 1b. & Passiflora Incarnata & .25 & 2.00 & & & & 5 \\
\hline & OZ. & Pueraria Thunbergiana & .35 & & & & & 1 \\
\hline & Ib. & $\begin{array}{l}\text { Tecoma Radicans } \\
\text { Vitis Coignetiae }\end{array}$ & $\begin{array}{l}.35 \\
.40\end{array}$ & 3.50 & 3.35 & & & $\begin{array}{r}29.25 \\
3.00\end{array}$ \\
\hline & " & Wistaria Multyuga, Blue & .40 & 4.00 & 3.85 & & & 33.75 \\
\hline & & FRUITS AND VEGETABLE & & & & & & \\
\hline & $"$ & Cherry, Mahaleb & .15 & 1.25 & 1.15 & & & 7.00 \\
\hline & $"$ & Maz zard & .15 & 1.25 & 1.15 & 1.10 & 1.00 & 75 \\
\hline & $"$ & Peach Pits, Chinese & 7 & 1.50 & 1.45 & & & \\
\hline & oz. & "Frereh & .00 & & & & & \\
\hline & Ib. & " Japan (Ussurjensis & & & & & & 3.0 \\
\hline & " & $\begin{array}{l}\text { Quince } \\
\text { Rhubarb }\end{array}$ & .35 & & & & & $\begin{array}{l}12.00 \\
.75\end{array}$ \\
\hline
\end{tabular}

5 Ib. 10 Ib. 25 Ib. per per prise prief paice tre

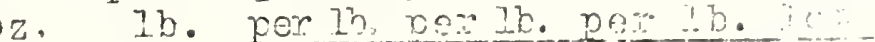
7 Ib. Rhus Copalina 4 " "Cotinus (1924 crop) $100 "$ Robinia Don-acacia .15 6 $\frac{1}{2}$ oz. Romneya Curiteri 1.00

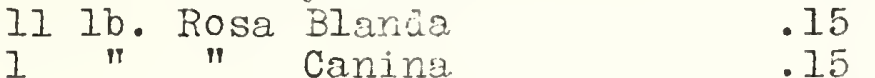
" Carojina .15 Setiorera .15 Salisburia Maiantifolia .15 Sambucus Candensis .15

.50

Sterculia Platanifolis $: 25$ shoricarpos Racemosus. 25

7.50

5.00

2.00

1.50

1.00

150

1. 50

$\begin{array}{ll}.35 & 1.25\end{array}$

351.25

.00

5.00

1.50

1.50

10.00

5.00

1.25

. 50

1.50

.50

.75

1.00

9.25

3.00

33.75

7.00

5.00

2.75

3.00

3.00

.75 
